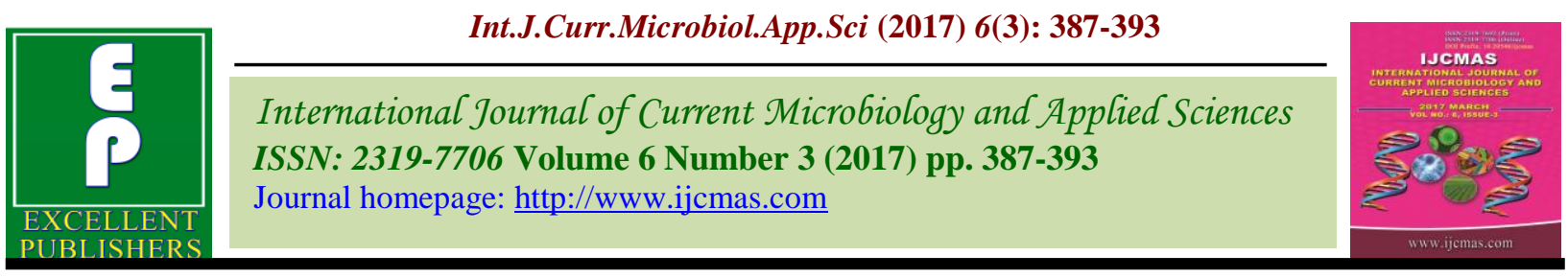

Original Research Article

https://doi.org/10.20546/ijcmas.2017.603.044

\title{
Heterosis in Snake Gourd (Trichosanthes cucumerina L.) for Growth and Earliness
}

\author{
N. Deepa Devi*, S. Mariappan and T. Arumugam \\ Department of Vegetable Science, Horticultural College and Research Institute, \\ Tamil Nadu Agricultural University, Coimbatore- 641003, India \\ *Corresponding author
}

\begin{tabular}{|c|c|}
\hline & A B S T R A C T \\
\hline Keywords & \multirow{4}{*}{ 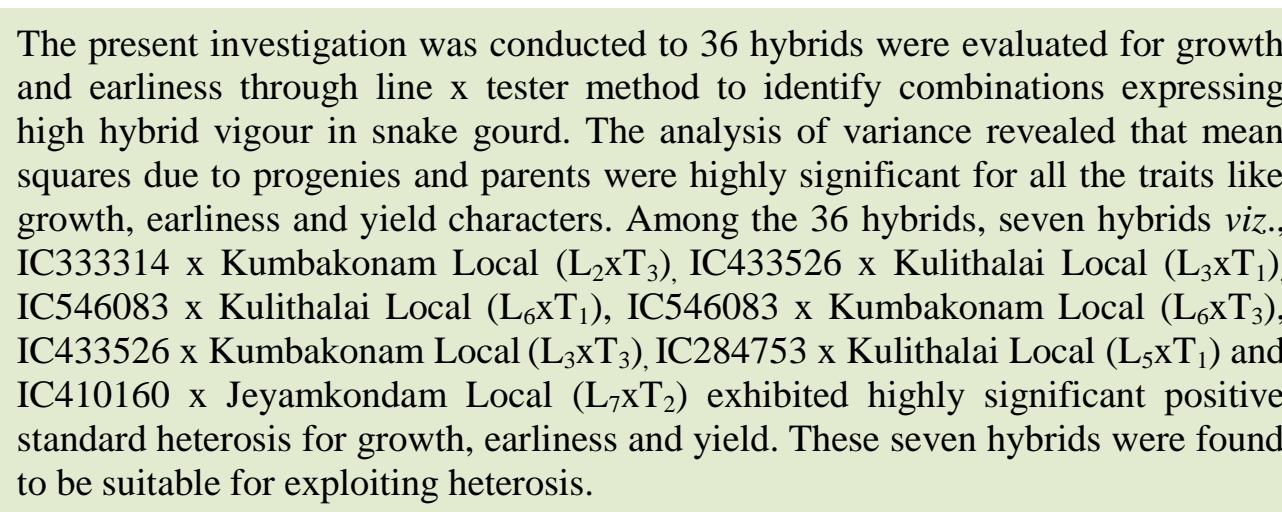 } \\
\hline $\begin{array}{l}\text { Snake gourd, } \\
\text { Trichosanthes } \\
\text { cucumerina L., } \\
\text { Line, Tester, } \\
\text { Heterosis. }\end{array}$ & \\
\hline Article Info & \\
\hline $\begin{array}{l}\text { Accepted: } \\
\text { 10 February } 2017 \\
\text { Available Online: } \\
\text { 10 March } 2017\end{array}$ & \\
\hline
\end{tabular}

\section{Introduction}

Snake gourd (Trichosanthes cucumerina L.) belongs to the family Cucurbitaceae and it's an important summer vegetable but it can be grown throughout the year except extreme winter. It is important as a good sources of minerals, fiber and nutrients to make the food wholesome and healthy (Ahmed et al., 2004). It is also one of the important vegetables which fetch more yield per unit area but the average yield of the crop is low. In addition it has got tremendous export potential because of its excellent keeping quality and shelf life. There are a number of cultivars with wide range of variability in size, shape and color of fruits available in this country. A large number of local lines are cultivated in the country but there is no recommended cultivar so far. There is no serious attempts have been made to upgrade the productivity of snake gourd. For developing superior varieties, it is necessary to improve the earliness and yield in snake gourd. This can be achieved through effective utilization of germplasm resources and integration of genomic tools to impart efficiency and pace of breeding processes (Banga, 2012). Exploitation of heterosis in crop plants is one of the most attractive achievements in boosting up the production and productivity of snake gourd. Heterosis breeding can be one of the most viable options for breaking the present yield barrier. Comprehensive analysis of the combining 
ability involved in the inheritance of quantitative traits and in the phenomenon of heterosis is necessary for evaluation of various breeding procedures (Allard, 1960 and Meena et al., 2015). However, the level of yield gain achieved from these hybrids is marginal. It clearly indicated that, the scope of improving the productivity of snake gourd through genetic manipulations. Keeping these points in view, the present investigation was undertaken to determine better parent heterosis of different cross combinations in snake gourd.

\section{Materials and Methods}

The present investigation was conducted in the Department of Horticulture, Agricultural College and Research Institute, Madurai, Tamil Nadu, during the period 2012-13. Study comprise of thirteen parents (nine lines and four testers) and these lines and testers (Table 1) were crossed in a LxT (Line $\mathrm{x}$ Tester) design to generate $36 \mathrm{~F}_{1}$ hybrids (crosses). These $36 \mathrm{~F}_{1}$ hybrids along with 13 parents were evaluated in randomized block design with three replications. These parents and hybrid seeds were sown at a spacing of $2 \mathrm{~m} \times 2 \mathrm{~m}$ with recommended package of practices followed as per the state of Tamil Nadu. Observations were recorded on five randomly tagged plants in each entry on vine length, internodal length, days to first female flower anthesis, days to first male flower anthesis, node at which first female flower appeared and fruit yield per vine. The data were analyzed for heterosis based on better parent was calculated following Mather and Jinks (1971).

\section{Results and Discussion}

The analysis of variance revealed that the parents and hybrids differed significantly for all the characters under study indicating the presence of considerable genetic variability among the genotypes. The magnitude of heterosis provides a basis for genetic diversity and a guide to the choice of parents for developing superior $F_{1}$ hybrids, so as to exploit hybrid vigor and or for building better gene pools to be employed in population improvement. The estimates of heterosis for growth, earliness and yield traits are presented in tables 2 and 3.

In this investigation, out of 36 hybrids, 18 hybrids showed significant positive heterosis and ten hybrids had significant negative heterosis over better parent for the trait vine length. The extent of heterosis over better parent was $-25.88\left(\mathrm{~L}_{9} \times \mathrm{T}_{3}\right)$ to $33.73\left(\mathrm{~L}_{8} \times \mathrm{T}_{2}\right)$ per cent. Among the 36 hybrids 14 hybrids showed significant positive heterosis over standard variety and the extent of heterosis over standard variety ranged between -38.54 $\left(\mathrm{L}_{9} \times \mathrm{T}_{3}\right)$ and $14.15\left(\mathrm{~L}_{2} \times \mathrm{T}_{3}\right)$ per cent. For the trait internodal length, 32 hybrids showed significant heterobeltiosis in negative direction (desirable). The magnitude of heterosis over better parent ranged between $30.08\left(\mathrm{~L}_{7} \mathrm{X} \mathrm{T}_{2}\right)$ and $7.66\left(\mathrm{~L}_{9} \mathrm{x} \mathrm{T}_{2}\right)$ per cent. The magnitude of heterosis over standard variety ranged from $-24.69\left(\mathrm{~L}_{9} \mathrm{x}_{3}\right)$ to 13.15 ( $\mathrm{L}_{9} \times \mathrm{T}_{2}$ ) per cent. As many as 30 hybrids showed heterosis in desirable direction over standard variety only five hybrids had significant positive heterosis over standard variety. For the trait days to first female flower anthesis, the negative heterobeltiosis estimates were significant in 32 hybrids. The highest significant negative value of -18.62 per cent was measured by the cross $\mathrm{L}_{1} \times \mathrm{T}_{2}$. Whereas the lowest value of 11.10 per cent was registered by $L_{9} \times T_{1}$. The highest significant negative standard heterosis was measured by the hybrid $\mathrm{L}_{1} \times \mathrm{T}_{2}$ (-9.25 per cent). Totally 21 hybrids showed significant negative heterosis and 11 exhibits significant positive heterosis over standard variety. The heterobeltiosis ranged from $-20.36\left(\mathrm{~L}_{1} \times \mathrm{T}_{2}\right)$ to $9.64\left(\mathrm{~L}_{2} \times \mathrm{T}_{2}\right)$ per cent were found in days to first male flower anthesis. Out of 36 hybrids, 31 hybrids showed significant 
negative heterosis over better parent. The magnitude of heterosis over standard variety was in the range of $-6.25\left(\mathrm{~L}_{7} \times \mathrm{T}_{3}\right)$ to 15.59 $\left(\mathrm{L}_{1} \mathrm{x} \mathrm{T}_{3}\right)$ per cent. Eight hybrids exhibited significant negative heterosis in desirable direction.

The negative heterobeltiosis estimates were significant in 30 hybrids was observed in the trait node at which first female flower appeared. The highest significant negative standard heterosis was measured by the hybrid $\mathrm{L}_{5} \times \mathrm{T}_{1}$ (-35.51 per cent). Totally 24 hybrids showed significant negative heterosis and seven exhibited significant positive heterosis over standard variety. For the trait yield per vine, the hybrid obtained from the cross $\mathrm{L}_{8} \times \mathrm{T}_{3}$ (141.39 per cent) registered the highest magnitude of heterosis in positive direction followed by $\mathrm{L}_{6} \times \mathrm{T}_{3}$ (139.27 per cent). Per cent heterosis of $F_{1} s$ over better parent value ranged $-108.31\left(\mathrm{~L}_{7} \mathrm{x} \mathrm{T}_{2}\right)$ to 133.00 per cent $\left(\mathrm{L}_{6} \times \mathrm{T}_{2}\right)$. Among them, twenty six crosses exhibited significant positive heterosis over better parent.

Table.1 Details of the parents used in the present study

\begin{tabular}{|c|l|l|l|}
\hline S. No. & \multicolumn{1}{|c|}{ Name of the parents } & \multicolumn{2}{|c|}{ Source } \\
\hline Lines & \multicolumn{2}{|l|}{} \\
\hline 1. & IC413017 & NBPGR, New Delhi & $\mathrm{L}_{1}$ \\
\hline 2. & IC333314 & NBPGR, New Delhi & $\mathrm{L}_{2}$ \\
\hline 3. & IC433526 & NBPGR, New Delhi & $\mathrm{L}_{3}$ \\
\hline 4. & IC308557 & NBPGR, New Delhi & $\mathrm{L}_{4}$ \\
\hline 5. & IC284753 & NBPGR, New Delhi & $\mathrm{L}_{5}$ \\
\hline 6. & IC546083 & NBPGR, New Delhi & $\mathrm{L}_{6}$ \\
\hline 7. & IC410160 & NBPGR, New Delhi & $\mathrm{L}_{7}$ \\
\hline 8. & IC202159 & NBPGR, New Delhi & $\mathrm{L}_{8}$ \\
\hline 9. & IC212527 & NBPGR, New Delhi & $\mathrm{L}_{9}$ \\
\hline Tester & & \multicolumn{2}{|l}{} \\
\hline 1. & Kulithalai Local & Tamil Nadu & $\mathrm{T}_{1}$ \\
\hline 2. & Jeyamkondam Local & Tamil Nadu & $\mathrm{T}_{2}$ \\
\hline 3. & Kumbakonam local & Tamil Nadu & $\mathrm{T}_{3}$ \\
\hline 4. & Palayajeyankondam Local & $\mathrm{T}_{4}$ \\
\hline
\end{tabular}


Table.2 Magnitude of heterosis (\%) over better parent for growth and earliness in snake gourd

\begin{tabular}{|c|c|c|c|c|c|c|}
\hline \multirow{2}{*}{ Hybrids } & \multicolumn{2}{|c|}{ Vine length $(\mathrm{cm})$} & \multicolumn{2}{|c|}{ Internode length } & \multicolumn{2}{|c|}{$\begin{array}{c}\text { Days to first female flower } \\
\text { anthesis }\end{array}$} \\
\hline & $\mathrm{BP}$ & SP & $\mathrm{BP}$ & SP & $\mathrm{BP}$ & SP \\
\hline $\mathbf{L}_{1} \times \mathbf{T}_{1}$ & $29.81 * *$ & -0.59 & $-14.86 * *$ & $-58.68 * *$ & $-31.69 * *$ & $11.47 * *$ \\
\hline $\mathbf{L}_{1} \times \mathbf{T}_{2}$ & 2.17 & $-17.27 * *$ & $47.61 * *$ & $-28.37 * *$ & $-38.46 * *$ & $-12.05 * *$ \\
\hline $\mathbf{L}_{1} \times \mathbf{T}_{3}$ & $23.29 * *$ & 2.24 & $0.02 \mathrm{NS}$ & $-51.46 * *$ & $-25.56 * *$ & $5.35 *$ \\
\hline $\mathbf{L}_{1} \times \mathbf{T}_{4}$ & $13.47 * *$ & $5.17 * *$ & $20.93 * *$ & $-41.32 * *$ & $-31.06 * *$ & $-8.83 * *$ \\
\hline $\mathbf{L}_{2} \times \mathbf{T}_{1}$ & $8.20 * *$ & $8.20 * *$ & $-36.68 * *$ & $-36.68 * *$ & $-22.80 * *$ & $25.97 * *$ \\
\hline $\mathbf{L}_{2 \times} \mathbf{T}_{2}$ & $11.12 * *$ & $11.12 * *$ & $-44.24 * *$ & $-44.24 * *$ & $-30.12 * *$ & $-0.13 \mathrm{NS}$ \\
\hline $\mathbf{L}_{2 \times} \mathbf{T}_{3}$ & $14.15 * *$ & $14.15 * *$ & $-60.97 * *$ & $-60.97 * *$ & $-12.59 * *$ & $23.71 * *$ \\
\hline $\mathbf{L}_{2 \times} \mathbf{T}_{4}$ & $-7.32 * *$ & $-7.32 * *$ & $-57.65 * *$ & $-57.65 * *$ & $-38.61 * *$ & $-18.81 * *$ \\
\hline $\mathbf{L}_{3} \times \mathbf{T}_{1}$ & $-20.87 * *$ & $-11.22 * *$ & $-38.52 * *$ & $-64.01 * *$ & $-61.90 * *$ & $-37.82 * *$ \\
\hline $\mathbf{L}_{3} \times \mathbf{T}_{2}$ & $-12.17 * *$ & -1.46 & $-30.19 * *$ & $-59.14 * *$ & $-29.49 * *$ & $4.70 \mathrm{NS}$ \\
\hline $\mathbf{L}_{3 \times} \mathbf{T}_{\mathbf{3}}$ & $-15.65 * *$ & $-5.37 * *$ & $-5.33 * *$ & $-44.58 * *$ & $-33.39 * *$ & $-1.10 \mathrm{NS}$ \\
\hline $\mathbf{L}_{3} \times \mathbf{T}_{4}$ & $-15.65 * *$ & $-5.37 * *$ & $29.23 * *$ & $-24.36 * *$ & $-45.98 * *$ & $-19.78 * *$ \\
\hline $\mathbf{L}_{4} \times \mathrm{T}_{1}$ & 1.80 & $10.24 * *$ & $40.44 * *$ & $-37.59 * *$ & $-53.00 * *$ & $-0.13 \mathrm{NS}$ \\
\hline $\mathbf{L}_{4} \times \mathbf{T}_{2}$ & 4.95 & $13.66 * *$ & $-28.68 * *$ & $-68.31 * *$ & $-43.91 * *$ & $19.20 * *$ \\
\hline $\mathbf{L}_{4} \times \mathbf{T}_{\mathbf{3}}$ & 0.00 & $8.29 * *$ & $19.04 * *$ & $-47.11 * *$ & $-43.91 * *$ & $19.20 * *$ \\
\hline $\mathbf{L}_{4} \times \mathbf{T}_{4}$ & $-16.22 * *$ & $-9.27 * *$ & $40.18 * *$ & $-36.50 * *$ & $-54.06 * *$ & $-2.38 \mathrm{NS}$ \\
\hline $\mathbf{L}_{5} \times \mathrm{T}_{1}$ & $-25.24 * *$ & $-24.88 * *$ & $2.37 * *$ & $-46.93 * *$ & $-35.83 * *$ & $4.70 \mathrm{NS}$ \\
\hline $\mathbf{L}_{5} \times \mathbf{T}_{2}$ & 1.94 & $2.44 *$ & $-32.46 * *$ & $-64.99 * *$ & $-34.40 * *$ & $-6.25 *$ \\
\hline $\mathbf{L}_{5} \times \mathbf{T}_{3}$ & -1.94 & -1.46 & $-31.13 * *$ & $-64.30 * *$ & $-25.11 * *$ & $5.99 *$ \\
\hline $\mathrm{L}_{5} \times \mathrm{T}_{4}$ & -5.83 & $-5.37 * *$ & $-2.28 * *$ & $-49.34 * *$ & $-24.48 * *$ & $-0.13 \mathrm{NS}$ \\
\hline $\mathrm{L}_{6} \times \mathrm{T}_{1}$ & $9.88 * *$ & $-13.17 * *$ & $-20.25 * *$ & $-29.23 * *$ & $-47.29 * *$ & $-13.98 * *$ \\
\hline $\mathbf{L}_{6} \times \mathrm{T}_{2}$ & $-16.87 * *$ & $-32.68 * *$ & $-51.76 * *$ & $-57.19 * *$ & $-2.61 \mathrm{NS}$ & $39.18 * *$ \\
\hline $\mathbf{L}_{6} \times T_{3}$ & $25.88 * *$ & $4.39 * *$ & $-5.53 * *$ & $-16.16 * *$ & $-34.44 * *$ & $-7.22 * *$ \\
\hline $\mathrm{L}_{6} \times \mathrm{T}_{4}$ & $16.84 * *$ & $8.29 * *$ & $-40.14 * *$ & $-46.88 * *$ & $-29.11 * *$ & $-6.25 *$ \\
\hline $\mathbf{L}_{7} \times \mathbf{T}_{1}$ & $31.21 * *$ & 0.49 & $-2.37 * *$ & $-50.37 * *$ & $-35.83 * *$ & 4.70 NS \\
\hline $\mathbf{L}_{7} \times \mathbf{T}_{2}$ & $31.33 * *$ & $6.34 * *$ & $24.35 * *$ & $-36.79 * *$ & $-20.42 * *$ & $13.72 * *$ \\
\hline $\mathbf{L}_{7} \times \mathbf{T}_{\mathbf{3}}$ & $7.06 * *$ & $-11.22 * *$ & $5.19 * *$ & $-46.53 * *$ & $-30.12 * *$ & $-1.10 \mathrm{NS}$ \\
\hline $\mathbf{L}_{7} \times \mathbf{T}_{4}$ & $8.42 * *$ & 0.49 & $-9.13 * *$ & $-53.81 * *$ & $-15.35 * *$ & $11.95 * *$ \\
\hline $\mathbf{L}_{8} \times \mathbf{T}_{1}$ & $8.28 * *$ & $-17.07 * *$ & $-10.77 * *$ & $-27.34 * *$ & $-30.50 * *$ & $13.40 * *$ \\
\hline $\mathbf{L}_{8} \times \mathbf{T}_{2}$ & $33.73 * *$ & $8.29 * *$ & $-22.03 * *$ & $-36.50 * *$ & $-25.83 * *$ & $5.99 *$ \\
\hline $\mathbf{L}_{8 \times} \mathbf{T}_{3}$ & -4.71 & $-20.98 * *$ & $-22.24 * *$ & $-36.68 * *$ & $-6.21 * *$ & $32.73 * *$ \\
\hline $\mathbf{L}_{8} \times \mathbf{T}_{4}$ & $21.05 * *$ & $12.20 * *$ & $6.26 * *$ & $-13.47 * *$ & $-34.96 * *$ & $-13.98 * *$ \\
\hline $\mathbf{L}_{9} \times T_{1}$ & $21.02 * *$ & $-7.32 * *$ & $3.91 * *$ & $-54.61 * *$ & $-34.25 * *$ & $7.28 * *$ \\
\hline $\mathbf{L}_{9} \times T_{2}$ & $-7.23 * *$ & $-24.88 * *$ & $-6.03 * *$ & $-62.87 * *$ & $-12.53 * *$ & $25.00 * *$ \\
\hline $\mathbf{L}_{9} \times T_{3}$ & $-25.88 * *$ & $-38.54 * *$ & $71.54 * *$ & $-24.01 * *$ & $-25.56 * *$ & $5.35 *$ \\
\hline $\mathbf{L}_{9} \times T_{4}$ & $18.95 * *$ & $10.24 * *$ & $9.69 * *$ & $-50.32 * *$ & $-15.23 * *$ & $12.11 * *$ \\
\hline
\end{tabular}


Table.3 Magnitude of heterosis (\%) over better parent for growth and earliness in snake gourd

\begin{tabular}{|c|c|c|c|c|c|c|}
\hline \multirow[t]{2}{*}{ Hybrids } & \multicolumn{2}{|c|}{$\begin{array}{c}\text { Days to first male flower } \\
\text { anthesis }\end{array}$} & \multicolumn{2}{|c|}{$\begin{array}{c}\text { Node at which first female } \\
\text { flower appeared }\end{array}$} & \multicolumn{2}{|c|}{ Yield per vine (kg) } \\
\hline & $\mathrm{BP}$ & SP & $\mathrm{BP}$ & $\mathrm{SP}$ & BP & SP \\
\hline $\mathbf{L}_{1 \times} \mathbf{T}_{1}$ & $-29.11 * *$ & $-22.32 * *$ & $-0.54 \mathrm{NS}$ & $-7.64 \mathrm{NS}$ & $-39.55 * *$ & $-20.35 * *$ \\
\hline $\mathbf{L}_{1 \times} \mathbf{T}_{2}$ & $-13.84 \mathrm{NS}$ & $-28.07 * *$ & $-3.31 \mathrm{NS}$ & $-10.21 * *$ & $-68.37 * *$ & $-19.48 * *$ \\
\hline $\mathbf{L}_{1 \times} \mathbf{T}_{3}$ & $-45.07 * *$ & $-34.29 * *$ & $-6.01 \mathrm{NS}$ & $-12.73 * *$ & $67.32 * *$ & $12.49 * *$ \\
\hline $\mathbf{L}_{1} \times \mathbf{T}_{4}$ & $-36.01 * *$ & $-16.10 *$ & $-9.03 * *$ & $-15.52 * *$ & $20.92 * *$ & $-16.69 * *$ \\
\hline $\mathbf{L}_{2 \times} \mathbf{T}_{1}$ & $-23.43 * *$ & $16.10 *$ & $54.07 * *$ & $54.07 * *$ & $-11.57 * *$ & $-11.57 * *$ \\
\hline $\mathbf{L}_{2 \times} \mathbf{T}_{2}$ & $-16.34 *$ & $-16.34 *$ & $5.39 \mathrm{NS}$ & $5.39 \mathrm{NS}$ & $27.79 * *$ & $27.79 * *$ \\
\hline $\mathbf{L}_{2} \times T_{3}$ & $4.95 \mathrm{NS}$ & $25.55 * *$ & $40.24 * *$ & $40.24 * *$ & $-12.48 * *$ & $-12.48 * *$ \\
\hline $\mathbf{L}_{2 \times} \mathbf{T}_{4}$ & $-31.63 * *$ & $-10.35 \mathrm{NS}$ & $37.25 * *$ & $37.25 * *$ & $24.10 * *$ & $24.10 * *$ \\
\hline $\mathbf{L}_{3 \times} \mathbf{T}_{1}$ & $-30.89 * *$ & $-0.78 \mathrm{NS}$ & $-3.31 \mathrm{NS}$ & $-10.21 * *$ & $9.93 * *$ & $6.60 * *$ \\
\hline $\mathbf{L}_{3} \times \mathbf{T}_{2}$ & $-29.22 * *$ & $1.62 \mathrm{NS}$ & $23.95 * *$ & $45.19 * *$ & $12.12 * *$ & $8.72 * *$ \\
\hline $\mathbf{L}_{3} \times \mathbf{T}_{\mathbf{3}}$ & $-16.55 * *$ & $19.81 * *$ & $97.78 * *$ & $20.68 * *$ & $32.01 * *$ & $28.01 * *$ \\
\hline $\mathbf{L}_{3 \times} \mathbf{T}_{4}$ & $-27.55 * *$ & $4.01 \mathrm{NS}$ & $94.11 * *$ & $18.44 * *$ & $-39.05 * *$ & $-0.89 \mathrm{NS}$ \\
\hline $\mathbf{L}_{4 \times} \mathbf{T}_{1}$ & $3.66 \mathrm{NS}$ & $13.58 *$ & $-15.61 * *$ & $-17.62 * *$ & $76.67 * *$ & $0.83 \mathrm{NS}$ \\
\hline $\mathbf{L}_{4} \times \mathbf{T}_{2}$ & $24.94 * *$ & $-10.35 \mathrm{NS}$ & $22.46 * *$ & $7.93 \mathrm{NS}$ & $106.10 * *$ & $43.84 * *$ \\
\hline $\mathbf{L}_{4} \times \mathbf{T}_{3}$ & $-19.86 * *$ & $-4.13 \mathrm{NS}$ & $25.63 * *$ & $10.73 *$ & $102.30 * *$ & $41.19 * *$ \\
\hline $\mathbf{L}_{4} \times \mathbf{T}_{4}$ & $-38.93 * *$ & $-19.93 * *$ & $-5.13 \mathrm{NS}$ & -7.39 & $59.35 * *$ & $49.35 * *$ \\
\hline $\mathbf{L}_{5 \times} \mathbf{T}_{1}$ & $-56.42 * *$ & $-52.24 * *$ & $22.86 * *$ & 7.95 & $87.21 * *$ & $45.37 * *$ \\
\hline $\mathbf{L}_{5} \times T_{2}$ & $-33.01 * *$ & $-34.29 * *$ & $87.11 * *$ & $39.54 * *$ & $122.83 * *$ & $47.40 * *$ \\
\hline $\mathbf{L}_{5} \times \mathbf{T}_{3}$ & $-40.07 * *$ & $-28.31 * *$ & $22.26 * *$ & $7.73 \mathrm{NS}$ & $113.89 * *$ & $0.38 \mathrm{NS}$ \\
\hline $\mathbf{L}_{5} \times \mathbf{T}_{4}$ & $-59.01 * *$ & $-46.26 * *$ & $101.00 * *$ & $49.90 * *$ & $46.23 * *$ & $37.06 * *$ \\
\hline $\mathrm{L}_{6} \times \mathrm{T}_{1}$ & $-53.82 * *$ & $-22.32 * *$ & $66.79 * *$ & $35.69 * *$ & $-71.19 * *$ & $-2.29 * *$ \\
\hline $\mathbf{L}_{6 \times} \mathrm{T}_{2}$ & $-46.71 * *$ & $-10.35 \mathrm{NS}$ & $65.73 * *$ & $34.83 * *$ & $133.00 * *$ & $0.55 \mathrm{NS}$ \\
\hline $\mathbf{L}_{6 \times} \mathbf{T}_{3}$ & $-43.01 * *$ & $-4.13 \mathrm{NS}$ & $62.23 * *$ & $31.98 * *$ & $125.74 * *$ & $51.77 * *$ \\
\hline $\mathrm{L}_{6} \times \mathrm{T}_{4}$ & $-39.59 * *$ & $1.62 \mathrm{NS}$ & $22.36 * *$ & $7.83 \mathrm{NS}$ & $41.63 * *$ & $-0.53 \mathrm{NS}$ \\
\hline $\mathbf{L}_{7} \times \mathbf{T}_{1}$ & $-11.63 \mathrm{NS}$ & $-3.17 \mathrm{NS}$ & $40.79 * *$ & $24.08 * *$ & $96.61 * *$ & $52.67 * *$ \\
\hline $\mathbf{L}_{7} \times \mathbf{T}_{2}$ & $-8.27 \mathrm{NS}$ & $-10.35 \mathrm{NS}$ & $36.52 * *$ & $20.32 * *$ & $-108.31 * *$ & $-10.10 * *$ \\
\hline $\mathbf{L}_{7} \times \mathbf{T}_{\mathbf{3}}$ & $-30.07 * *$ & $-16.34 *$ & $32.65 * *$ & $16.92 * *$ & $103.11 * *$ & $0.37 \mathrm{NS}$ \\
\hline $\mathbf{L}_{7} \times \mathbf{T}_{4}$ & $-38.93 * *$ & $-19.93 * *$ & $30.92 * *$ & $15.39 * *$ & $49.73 * *$ & $40.34 * *$ \\
\hline $\mathbf{L}_{8 \times} \mathbf{T}_{1}$ & $-45.71 * *$ & $-22.32 * *$ & $-0.71 \mathrm{NS}$ & $-21.99 * *$ & $50.14 * *$ & $16.58 * *$ \\
\hline $\mathbf{L}_{8 \times} \mathbf{T}_{2}$ & $-54.08 * *$ & $-34.29 * *$ & $0.39 \mathrm{NS}$ & $-13.95 * *$ & $97.58 * *$ & $22.15 * *$ \\
\hline $\mathbf{L}_{8 \times} T_{3}$ & $4.48 \mathrm{NS}$ & $49.49 * *$ & $33.30 * *$ & $35.56 * *$ & $113.89 * *$ & $43.80 * *$ \\
\hline $\mathbf{L}_{8} \times T_{4}$ & $3.64 \mathrm{NS}$ & $48.29 * *$ & $34.90 * *$ & $37.19 * *$ & $49.12 * *$ & $39.76 * *$ \\
\hline $\mathbf{L}_{9} \mathrm{x} \mathbf{T}_{1}$ & $-68.99 * *$ & $-40.04 * *$ & $63.88 * *$ & $6.42 \mathrm{NS}$ & $-75.12 * *$ & $-0.05 \mathrm{NS}$ \\
\hline $\mathbf{L}_{9} \mathbf{x} \mathbf{T}_{2}$ & $-52.27 * *$ & $-7.72 \mathrm{NS}$ & $63.12 * *$ & $5.92 \mathrm{NS}$ & $-85.62 * *$ & $-10.69 * *$ \\
\hline $\mathbf{L}_{9 \times} \mathbf{T}_{3}$ & $-53.64 * *$ & $-10.35 \mathrm{NS}$ & $52.92 * *$ & $29.59 * *$ & $80.13 * *$ & $24.15 * *$ \\
\hline $\mathbf{L}_{9} \times T_{4}$ & $-47.45 * *$ & $1.62 \mathrm{NS}$ & $-15.31 * *$ & $-17.32 * *$ & $-27.96 * *$ & $-16.28 * *$ \\
\hline
\end{tabular}


The hybrid obtained from the cross $L_{6} \times T_{2}$ (133.00 per cent) registered the highest magnitude of heterosis in positive direction followed by $\mathrm{L}_{6} \times \mathrm{T}_{3}(125.74)$.

The important criterion to assess the hybrids for heterosis breeding was through better parent (Heterobeltiosis) and standard variety (Standard heterosis). Though, the heterosis are important, Kadambavana Sundaram (1980) suggested that the heterotic expression over standard variety should alone be given due importance for commercial exploitation of hybrid vigour. Hence, the crosses, which showed significantly high value of standard heterosis over Kumbakonam Local $\left(\mathrm{T}_{3}\right)$ followed by Kulithalai Local $\left(\mathrm{T}_{1}\right)$ for growth and earliness traits are taken into account. Significant heterosis over standard variety was observed for all the growth, earliness and yield traits in $\mathrm{L}_{2} \mathrm{xT}_{3}, \mathrm{~L}_{3} \mathrm{xT}_{1}, \mathrm{~L}_{6} \mathrm{xT}_{1}$ and $\mathrm{L}_{6} \mathrm{xT}_{3}$.

The next best hybrids were $\mathrm{L}_{3} \mathrm{xT}_{3}, \mathrm{~L}_{5} \mathrm{xT}_{1}$ and $\mathrm{L}_{7} \mathrm{xT}_{2}$ expressed significantly high standard heterosis for all the traits. From this discussion, it is clear that the above said hybrids are highly suitable for heterosis breeding. The similar findings were reported by Banik (2003) and Rahman (2004) in snake gourd, Dubey and Maurya (2007) and Suganthi (2008) in bottle gourd, Sarkar and Sirohi (2010) in cucumber, Podder (2010) in snake gourd, Vegad et al., (2011) in bottle gourd, Alli Rani (2013) and Narasannavar (2014) in ridge gourd and Meena (2015) in Indian mustard.

In conclusion, the hybrids $\mathrm{L}_{2} \mathrm{xT}_{3}, \mathrm{~L}_{3} \mathrm{xT}_{1}$, $\mathrm{L}_{6} \mathrm{xT}_{1}, \mathrm{~L}_{6} \mathrm{xT}_{3}, \mathrm{~L}_{3} \mathrm{xT}_{3}, \mathrm{~L}_{5} \mathrm{xT}_{1}$ and $\mathrm{L}_{7} \mathrm{xT}_{2}$ which recorded high heterotic values for growth, earliness and yield. Hence, these crosses could be utilized successfully in further breeding programmes for exploitation of heterosis and developing desirable genotypes for improving yield parameters.

\section{Acknowledgement}

I am very grateful to the University Grants Commission (UGC), New Delhi for providing Fellowship to my Ph.D programme and also thankful to the Director, NBPGR, New Delhi for supplying the seeds of different snake gourd germplasm.

\section{References}

Ahmed, A.M., Reddy, T.P. and Neeraja, G. 2004. Heterosis for fruit yield and yield components in Ridge Gourd [Luffa acutangula (Roxb.) L.]. J. Res. Angrau., 34(1): 15-20.

Allard, R.W. 1960. Principles of plant Breeding. John Wiley and Sons. Inc., U.S.A. p.485.

Alli Rani, E. 2013. Genetic studies on growth, yield and mosaic tolerance in Ridge Gourd [Luffa actuangula L. (Roxb)]. M.Sc., (Hort.) Thesis, Tamil Nadu Agricultural University, Coimbatore.

Banga, S.S. 2012. Germplasm Enhancement in Indian Mustard: Some Exiting New Developments. In: "Souvenir of XIX Annual AICRP Group Meet on Rapeseed-Mustard", Birsa Agricultural University, Ranchi, India, PP. 29-34.

Banik, B.R. 2003. Variability, gene action and heterosis in snakegourd (Trichosanthes anguina L.). Ph. D. Dissertation. Department of Genetics and Plant Breeding, BSMRAU, Salna, Gazipur, Bangladesh.

Dubey, S.K. and Maurya, I.B. 2007. Combining ability for character related to yield and earliness in bottle gourd [Lagenaria siceraria (Mol.) Standl.]. Indian J. Agri. Res., 4(1): 59-62.

Kadambavanasundaram, M. 1980. Heterotic system in cultivated species of Gossypium. An appraisal (Abst). Genetic and crop improvement of heterotic systems. In: Pre-congress 
scientific meeting of $\mathrm{XV}$ international congress of genetics, TNAU, Coimbatore, pp.20.

Mather, K. and Jinks, J.L. 1971. Biometrical Genetics. Chapman and Hall, New York.

Meena, H.S., Kumar, A. Ram, B., Singh, V.V., Meena, P.D., Singh, B.K. and Singh, D. 2015. Combining Ability and Heterosis for Seed Yield and Its Components in Indian Mustard (Brassica juncea L.). J. Agr. Sci. Tech., 17: 1861-1871.

Narasannavar, A.R., Gasti, V.D., Shantappa, T., Mulge, R., Allolli, T.B. and Thammaiah, N. 2014. Heterosis studies in ridge gourd [Luffa acutangula (L.) Roxb.] Karnataka J. Agric. Sci., 27(1): 47-51.

Podder, R., Rasul, M.G., Islam, A.K.M.A., Mian, M.A.K. and Ahmed, J.U. 2010. Combining Ability and Heterosis in
Snake gourd (Tricosanthes cucurminata L). Bangladesh J. Pl. Breed. Genet., 23(2): 01-06.

Rahman, A.K.M.M. 2004. Genotypeenvironment interaction, heterosis and sex modification in snakegourd (Trichosanthes anguina L.). Ph. D. Department of Genetics and Plant Breeding, BSMRAU, Salna, Gazipur, Bangladesh.

Sarkar, M. and Sirohi, P.S. 2010. Combining ability analysis for yield and its attributing character in Cucumber. Indian J. Hort., 67(4): 525-530.

Suganthi, M., 2008. LxT analysis in Bottle Gourd [Lagenaria siceraria (Mol.) Stand]. M.Sc. Thesis. Tamil Nadu Agricultural University, Coimbatore.

Vegad, P.M., Vaddoria, M.A., Mehta, D.R. and Naghera, Y.V. 2011. Combining ability analysis in Bottle Gourd. Crop Improvement, 38(1): 13-17.

\section{How to cite this article:}

Deepa Devi, N., S. Mariappan and Arumugam, T. 2017. Heterosis in Snake Gourd (Trichosanthes cucumerina L.) for Growth and Earliness. Int.J.Curr.Microbiol.App.Sci. 6(3): 387-393. doi: https://doi.org/10.20546/ijcmas.2017.603.044 